\title{
Evaluación clínica de la salud oral de niños con neoplasias malignas
}

\author{
Gordón-Núñez MA*, Pereira Pinto L**, Souza BL***, \\ Oliveira PT****, Fernandes MZ******
}

\section{RESUMEN}

Objetivo: Éste estudio de carácter descriptivo visó evaluar clínicamente niños con neoplasias malignas y poner de manifiesto la relación entre el status de salud bucal y la ocurrencia de complicaciones estomatológicas.

Pacientes y métodos: la salud bucal de 40 niños con neoplasias malignas (grupo I) fue evaluada clínicamente mediante examen físico extra e intra oral, obtención del índice de placa visible, índice de sangrado gingival e índice de dientes cariados, ausentes y obturados y simultáneamente fueron observadas las complicaciones estomatológicas desarrolladas en esos pacientes. El status de salud bucal de éstos niños fue comparado al de niños saludables (grupo II).

Resultados: los niños del grupo I presentaron índices de sangrado gingival y experiencia de caries menores que los del grupo II, por otro lado, el índice de placa visible fue ligeramente mayor en los niños del grupo I, sin embargo, sólo hubo diferencia estadísticamente significativa en el valor del CAO-D/cao-d entre ambos grupos ( $\mathrm{p}=$ 0.002). En el grupo I, 16 niños desarrollaron conjuntamente 61 complicaciones estomatológicas, con predominancia de la mucositis, seguida del sangrado oral espontáneo, candidiasis y xerostomia.

Conclusión: Pacientes bajo tratamiento antineoplásico, presentando higiene oral deficiente, tienen mayor riesgo de desarrollar complicaciones estomatológicas.

Palabras clave: neoplasias malignas, terapia antineoplásica, salud bucal, complicaciones estomatológicas.

\section{ABSTRACT}

Objective: In this descriptive study were assessment clinically children with malignant neoplasm, to evidence the correlation between oral health status and the occurrence of stomatologic complications.

Patients and methods: Were evaluated the oral health conditions of 40 children with malignant neoplasm (group I) through intra and extra oral physic examination, obstention of Visible Plate Index, Gingival Bleeding Index and Decayed, Missed and Filled Teeth Index; simultaneously were observed the stomatologic complications developed in this patients. The oral health status of the children of group I was compared to the healthy children (group II). Results: The children of group I presented Gingival Bleeding Index and caries experience lower than children of group II, in other hand, the Visible Plate Index was lightly greater in children of group I, nevertheless existed statistical difference in the value of the DMF-T/dmf-t between both groups $(p=0.002)$. In the group I, 16 children developed together 61 stomatologic complications, with predominance of oral mucositis, followed by spontaneous oral bleeding, candidiasis and xerostomy.

Conclusions: Patients in anti neoplastic therapy with deficient oral hygiene had high risk of develop stomatologic complications.

Key words: Malignant neoplasm, antineoplastic therapy, oral health, stomatologic complications.

Aceptado para publicación: Septiembre 2004. 
* $\quad$ Alumno de Doctorado en Patología Oral - UFRN.

** Dr. Prof. Titular del Programa de Post Grado en Patología Oral - UFRN.

*** Prof. Dra. del Programa de Post Grado en Patología Oral - UFRN.

**** Dra. en Estomatología.

***** Especialista en Pediatría y Hematología Clínica - Prof. de la Facultad de Medicina - UFRN.

Estudio financiado por la Coordenação de Aperfeiçoamento de Pessoal de Nivel Superior-CAPES-Brasil.

Gordón-Núñez MA, Pereira Pinto L, Souza BL, Oliveira PT, Fernandes MZ. Evaluación clínica de la salud oral de niños con neoplasias malignas. Av. Odontoestomatol 2005; 21-3: 127-139.

\section{IINTRODUCCIÓN}

Las neoplasias malignas constituyen la tercera causa de muerte en humanos de todo el mundo incluyendo los pacientes pediátricos. El propio curso clínico de las neoplasias, aliado a los agentes terapéuticos antineoplásicos pueden generar complicaciones orgánicas, incluso en la cavidad oral, con constantes ocurrencias.

En la infancia el cáncer generalmente acomete estructuras predominantemente embrionarias con disturbios en la proliferación y diferenciación celular, comprometiendo, con mayor frecuencia, las células del sistema hematopoyético, constituyendo así, las denominadas neoplasias sistémicas. Entre las neoplasias sistémicas, las leucemias se colocan en primer lugar, seguidas de los linfomas (1), sin embargo, se observa también, grande ocurrencia de tumores sólidos (2).

Las complicaciones estomatológicas que ocurren en niños oncológicos pueden ir desde mucositis, infecciones oportunistas, xerostomia y enfermedad periodontal hasta las alteraciones en el desarrollo de los dientes y maxilares. Ellas pueden contribuir con el agravamiento del cuadro clínico del paciente y comprometer la respuesta inmunológica, aumentando significativamente, el grado de morbidad y mortalidad de los pacientes. Muchas de esas alteraciones orales pueden constituir la primera señal del desarrollo de neoplasias malignas, principalmente en el caso de las leucemias (1).

La terapia utilizada en el tratamiento del cáncer, específicamente la quimioterapia y la radioterapia pueden constituir agentes potencialmente inducto- res de complicaciones estomatológicas; además de ellas, integran el elenco de factores asociados a esas complicaciones el desequilibrio de la microbiota oral, la higiene bucal deficiente, enfermedades ó alteraciones orales preexistentes y la presencia de trauma local.

Como consecuencia del comprometimiento inmunológico del paciente, la cavidad oral se torna un ambiente ideal para la colonización y proliferación de microorganismos capaces de promover no solo infecciones oportunistas, sino también, cuadros severos de septicemia; sobresaliendo entre ellas las infecciones fúngicas, principalmente por Candida $\operatorname{spp}(3)$.

Los estados trombocitopénicos provocados por neoplasias malignas, la quimioterapia y/ó radioterapia pueden conducir a eventos de sangrado oral espontáneo, sin embargo, otros factores pueden ser considerados como causa de sangrado oral en éstos pacientes, tales como alteraciones en el sistema de coagulación sanguínea, enfermedades de base y lesiones orales pre existentes $(4,5)$.

Los procesos cariosos en pacientes oncológicos pueden guardar relación con el estado inmunológico comprometido, la presencia de xerostomia y el desequilibro de la microbiota oral, factores éstos, que favorecen la proliferación de microorganismos cariogénicos (6).

En éste trabajo se objetiva realizar una evaluación clínica de las condiciones de salud oral de niños con neoplasias malignas atendidos en el Centro de Oncología y Hematología Infantil (COHI) del Hospital Infantil Varela Santiago (HISV) de Natal / RN, Brasil y 
simultáneamente registrar las complicaciones estomatológicas constatadas en esos pacientes.

\section{PACIENTES Y MÉTODOS}

La muestra fue constituida de 78 niños con edades oscilando de los 0 a 15 años, divididos en dos grupos. El grupo I estuvo formado por 40 niños con neoplasias malignas, divididos en dos subgrupos, siendo uno constituido de niños con neoplasias sistémicas y el otro de niños con tumores sólidos bajo tratamiento en el COHI del HIVS. El grupo II incluyó 38 niños saludables, con edades y sexos iguales a los del grupo I, alumnos del Centro de Atención Integral del Niño y del Adolescente (CAIC) de la Ciudad Satélite en Natal / RN - Brasil. Éste grupo fue utilizado comparar su status de salud oral con el de los niños oncológicos.

Los pacientes oncológicos, al inicio del estudio fueron sometidos a anamnesia, la cual fue respondida con la ayuda de un adulto responsable, seguidamente se realizó el examen físico intra y extra oral. El examen intra oral constó de inspección visual y palpación digital de las estructuras bucales. La encía fue evaluada mediante la obtención del índice de sangrado gingival (ISG) de Ainamo y Bay (7) y los dientes con los índices de placa visible (IPV) $(8,9)$, el cual constituye una variante del Índice de Placa de Silness $\varepsilon$ Löe, que permite verificar fácilmente la capacidad de control de la biopelícula por el paciente, debido a que a través de él se considera la presencia ó no de biopelícula sobre los dientes. Según éste índice, las superficies dentarias que presentan placa visible reciben un valor $(0=\sin$ placa, $1=$ con placa) los valores positivos son sumados y el resultado es dividido por el número de superficies evaluadas, siendo finalmente el resultado multiplicado por 100 para expresar el IPV del paciente en porcentaje; también formó parte de ésta evaluación la obtención del índice de dientes cariados, ausentes y obturados (CAO-D/cao-d).

Se realizaron en media 2 evaluaciones semanales en cada niño, durante el periodo de 7 meses, en cada visita del niño al $\mathrm{COHI}$ para las sesiones de quimioterapia, visitas ambulatoriales de control clínico ó durante el período de internamiento en el centro antes mencionado. Los datos personales e informaciones del tipo de oncología, tratamiento y el recuento plaquetario de los niños oncológicos fueron obtenidos de los expedientes clínicos. En los pacientes saludables fueron realizados los mismos procedimientos y la misma cantidad de evaluaciones en visitas periódicas al CAIC. La evaluación de las condiciones de salud bucal de los dos grupos fue realizada complementando los datos del ISG con los obtenidos mediante la aplicación del IPV. Éste análisis se basa en el hecho de que el ISG representa la presencia de biopelícula envejecida, con subsiguiente compromiso gingival y el IPV, se refiere a la presencia de biopelícula joven que no fue capaz de desarrollar inflamación gingival hasta el momento del examen.

El diagnóstico de candidiasis fue realizado por el aspecto clínico de las lesiones de acuerdo con los criterios de la EC-CLEARINGHOUSE (10) y por la respuesta al tratamiento con nistatina. La xerostomia fue caracterizada subjetivamente por el aspecto reseco de la mucosa oral. El sangrado oral espontáneo fue diagnosticado por la presencia de sangrado en cualquier lugar de la mucosa oral que ocurriera sin causa aparente; resultados dos exámenes hematológicos referentes al recuento de plaquetas fueron colectados semanalmente y valores menores a 100.000 células $/ \mathrm{mm}^{3}$ fueron considerados factor de riesgo para la ocurrencia de sangrado oral espontáneo (11).

El diagnostico de mucositis fue establecido mediante la observación de los criterios definidos por Epstein y cols.(12). Las informaciones colectadas fueron registradas en la ficha clínica y sometidas al análisis estadístico con el test exacto de Fisher y con el test "t" de student no pareado, considerando significativas diferencias $<0.05$. En el análisis estadísticos de los datos del grupo I, el Odds Ratio fue utilizado como medida de asociación entre las variables (tipo de neoplasia e complicaciones estomatológicas) y cuando era verificada esa asociación se utilizó el riesgo relativo (RR) para saber si alguno de los subgrupos de niños oncológicos (neoplasias sistémicas / tumores sólidos) presentaba mayor riesgo de desarrollar una determinada complicación estomatológica. 
Éste trabajo fue aprobado por el Comité de Ética en la Investigación de la Universidad Federal do Rio Grande do Norte (UFRN) y del HIVS; los padres ó responsables por los niños fueron informados del carácter y objetivos de la investigación, expresando su consentimiento a través de la firma de un documento de autorización informada.

\section{RESULTADOS}

Del total de 78 niños evaluados en los dos grupos, $34(43.6 \%)$ eran del sexo femenino y $44(56.4 \%)$ del sexo masculino. Los 40 niños del grupo I se encontraban entre las edades de 02 meses a 15 años, de los cuales 18 (45\%) eran del sexo femenino y 22 (55 $\%)$ del masculino.

En el grupo I, fueron diagnosticados 25 niños (62.5 $\%)$ con neoplasias sistémicas y 15 (37.5\%) con tumores sólidos (Cuadro 1).
El grupo II era constituido de 38 niños entre las edades de 6 meses a 15 años, de los cuales, 16 (42.1\%) eran del sexo femenino y $22(57.9 \%)$ del sexo masculino.

En ambos grupos la frecuencia de cepillado varió de 1 a 3 veces al día, con media de 2 cepillados diarios; no se observaron diferencias estadísticamente significativas en el número de cepillados entre los niños oncológicos y los sanos. El IPV fue obtenido en 38 niños del grupo I, de los cuales, 19 (50\%) tuvieron bajo IPV y 19 (50 \%) alto IPV. En el grupo II, fueron evaluados 37 niños con éste índice, siendo que, 19 (51.4 \%) presentaron bajo IPV y 18 (48.6\%) alto IPV. El test $\mathrm{t}$ de student no pareado no mostró diferencia estadísticamente significativa en el valor del IPV entre ambos grupos $(\mathrm{p}=0.86)$ (Tabla 1$)$.

El índice de sangrado gingival (ISG) fue obtenido en 36 niños del grupo I, de los cuales, 28 (77.7 \%) tuvieron bajo ISG y 8 (22.3 \%) alto ISG. El ISG fue obtenido en 36 niños del grupo II, de los cuales, 26 (70.3

\begin{tabular}{|c|c|c|c|c|c|}
\hline \multicolumn{6}{|c|}{$\begin{array}{l}\text { CUADRO 1.- DISTRIBUCIÓN DE LOS NIÑOS DEL GRUPO I CON RELACIÓN AL SEXO, } \\
\text { EDAD Y TIPO DE NEOPLÁSIA. NATAL/RN-2001 }\end{array}$} \\
\hline \multicolumn{6}{|c|}{ Neoplasias sistémicas } \\
\hline Neoplasia & $n^{\circ}$ de casos & $\%$ & Sexo & Rango de edades & Edad media \\
\hline $\begin{array}{l}\text { LLA * } \\
\text { LMA } \dagger \\
\text { D. de Hodgkin } \\
\text { L. Burkitt } \\
\text { LNH - cel. B } \ddagger\end{array}$ & $\begin{array}{l}13 \\
8 \\
2 \\
1 \\
1\end{array}$ & $\begin{array}{c}52.0 \\
32.0 \\
8.0 \\
4.0 \\
4.0\end{array}$ & $\begin{array}{c}6 F-7 M \\
5 F-3 M \\
1 F-1 M \\
F \\
M\end{array}$ & $\begin{array}{c}2 \text { meses }-14 \text { años } \\
8 \text { meses }-13 \text { años } \\
10 \text { años }-12 \text { años } \\
4 \text { años } \\
6 \text { años }\end{array}$ & $\begin{array}{l}5.9 \text { años } \\
7.2 \text { años } \\
11 \text { años } \\
4 \text { años } \\
6 \text { años }\end{array}$ \\
\hline \multicolumn{6}{|c|}{ Tumores sólidos } \\
\hline Neoplasia & $n^{\circ}$ de casos & $\%$ & Sexo & Rango de edades & Edad media \\
\hline $\begin{array}{l}\text { Neuroblastoma } \\
\text { Tumor de Wilms } \\
\text { Glioblastoma Maligno } \\
\text { Rabdomiosarcoma } \\
\text { Leiomiosarcoma } \\
\text { Fibrosarcoma } \\
\text { PNET } §\end{array}$ & $\begin{array}{l}5 \\
4 \\
2 \\
1 \\
1 \\
1 \\
1\end{array}$ & $\begin{array}{l}33.3 \\
26.7 \\
13.3 \\
6.7 \\
6.7 \\
6.7 \\
6.7\end{array}$ & $\begin{array}{c}1 F-4 M \\
3 F-1 M \\
2 M \\
F \\
M \\
M \\
M\end{array}$ & $\begin{array}{c}6 \text { meses }-5 \text { años } \\
1 \text { año - } 15 \text { años } \\
8 \text { años - } 13 \text { años } \\
4 \text { años } \\
6 \text { años } \\
8 \text { años } \\
13 \text { años }\end{array}$ & $\begin{array}{l}3.3 \text { años } \\
5.7 \text { años } \\
10.5 \text { años } \\
4 \text { años } \\
6 \text { años } \\
8 \text { años } \\
13 \text { años }\end{array}$ \\
\hline $\begin{array}{l}\text { Fuente: Centro de Oncología } \\
\text { * LLA: Leucemia Linfoblástica } \\
\text { § PNET: Tumor Neuroectodérm }\end{array}$ & $\begin{array}{l}\text { matología Infa } \\
\text { ıda; † LLA: Leı } \\
\text { de la Infancia. }\end{array}$ & HIV & $\begin{array}{l}\text { al } R N-2001 \\
\text { Aguda; } \ddagger L N\end{array}$ & a no Holding de Célul. & \\
\hline
\end{tabular}


TABLA 1.- MEDIA, DESVÍO PATRÓN, INTERVALO DE CONFIANZA Y VALOR DE P PARA EL IPV DE LA MUESTRA EVALUADA. NATAL / RN-2001

\begin{tabular}{|lccccc|}
\hline \multicolumn{1}{r}{ Grupo } & $\boldsymbol{n}^{\text {o }}$ & Media & DP & IC (95\%) & $p$ \\
\hline GRUPO I & 38 & 29.57 & 32.60 & $18.85-40.29$ & 0.86 \\
\hline GRUPO II & 37 & 28.35 & 27.57 & $19.15-37.55$ & - \\
\hline Fuente: Centro de Oncología y Hematología Infantil del HIVS. Natal / RN - 2001 & & \\
\hline
\end{tabular}

TABLA 2.- MEDIA, DESVÍO PATRÓN, INTERVALO DE CONFIANZA Y VALOR DE P PARA EL ISG DE LA MUESTRA EVALUADA. NATAL / RN-2001

\begin{tabular}{|lccccc|}
\hline \multicolumn{1}{|c}{ Grupo } & $\boldsymbol{n}^{\circ}$ & Media & DP & IC (95\%) & $p$ \\
\hline GRUPO I & 36 & 1.31 & 3.02 & $0.29-2.33$ & 0.12 \\
\hline GRUPO II & 36 & 2.70 & 4.44 & $1.21-4.19$ & - \\
\hline Fuente: Centro de Oncología y Hematología Infantil del HIVS. Natal / RN - 2001 & & \\
\hline
\end{tabular}

\section{TABLA 3.- MEDIA, DESVÍO PATRÓN, INTERVALO DE CONFIANZA Y VALOR DE P PARA EL ISG} DE LA MUESTRA EVALUADA. NATAL / RN-2001

\begin{tabular}{|lccccc|}
\hline \multicolumn{1}{|c}{ Grupo } & $\boldsymbol{n}^{\mathbf{0}}$ & Media & DP & IC (95\%) & $p$ \\
\hline GRUPO I & 38 & 2.13 & 2.61 & $0.27-2.99$ & 0.12 \\
\hline GRUPO II & 37 & 4.54 & 3.82 & $3.27-5.81$ & - \\
\hline Fuente: Centro de Oncología y Hematología Infantil del HIVS. Natal / RN - 2001 & & \\
\hline
\end{tabular}

\%) tuvieron bajo ISG y 11 (29.7 \%) alto ISG. El test t de student no pareado no mostró diferencia estadísticamente significativa en el ISG entre los grupos I y II $(p=0.12)$ (Tabla 2).

El índice CAO-D/cao-d fue obtenido en 38 niños del grupo I. En éstos, el número de dientes cariados por paciente varió de 0 a 7 , el número de dientes ausen- tes fue de 0 a 6 y el número de dientes obturados fue de 0 a 4 . En el grupo II ese índice fue obtenido en 37 niños, éstos presentaron de 0 a 12 dientes cariados, de 0 a 6 dientes ausentes y de 0 a 2 dientes obturados. El test t de student no pareado mostró diferencia estadísticamente significativa en el CAO-D/cao-d entre los grupos evaluados ( $\mathrm{P}=$ 0.002) (Tabla 3).

$\square$ Mucositis
$\square$ Candidiasis
a Sangrado oral espontáneo
$\square$ Xerostomia
$\square$ Otras lesiones

Figura 1. Distribución de los niños con neoplasias malignas con complicaciones orales en el grupo I. Natal /RH-2001. 


\begin{tabular}{|c|c|c|c|c|}
\hline \multicolumn{5}{|c|}{$\begin{array}{c}\text { CUADRO 2.- DISTRIBUCIÓN DE LOS NIÑOS CON SUS RESPECTIVOS EPISODIOS DE } \\
\text { CANDIDIASIS EN EL GRUPO I, CON RELACIÓN A LA EDAD, SEXO Y EL TIPO DE NEOPLASIA. } \\
\text { NATAL / RN-2001 }\end{array}$} \\
\hline Caso & Edad & Sexo & Neoplasia & Tipo y localización de los episodios de candidiasis \\
\hline 18 & $09 \mathrm{a}$ & $\mathrm{F}$ & LMA & Pseudomembranosa - región vestibular de la encía adherida inferior. \\
\hline 19 & $02 \mathrm{~m}$ & $\mathrm{~F}$ & LLA & Pseudomembranosa - suelo de la boca y mucosa de carrillos \\
\hline 19 & $02 \mathrm{~m}$ & $\mathrm{~F}$ & LLA & Queilitis angular \\
\hline 22 & $07 \mathrm{a}$ & $\mathrm{F}$ & LMA & Pseudomembranosa - dorso de la lengua \\
\hline 22 & $07 \mathrm{a}$ & $\mathrm{F}$ & LMA & Pseudomembranosa - mucosa de carrillos \\
\hline 22 & 07 a & $\mathrm{F}$ & LMA & Queilitis angular \\
\hline 22 & $07 \mathrm{a}$ & $\mathrm{F}$ & LMA & Queilitis angular \\
\hline 23 & $11 \mathrm{a}$ & $\mathrm{F}$ & LLA & Pseudomembranosa - mucosa de carrillos \\
\hline 31 & $12 \mathrm{a}$ & $\mathrm{F}$ & D. $\mathrm{H}$ & Pseudomembranosa - mucosa de carrillos \\
\hline
\end{tabular}

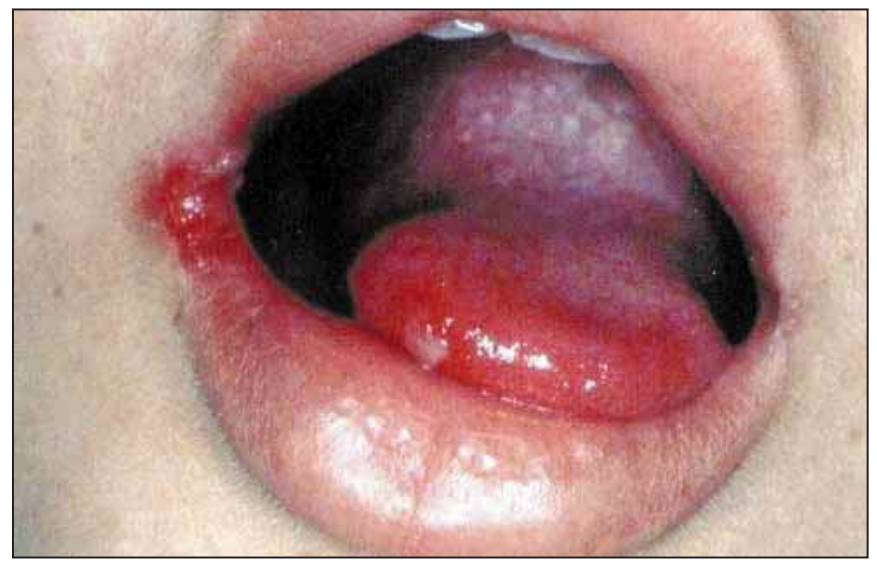

Figura 7. Paciente con LLA presentando queilitis angular, candidiasis pseudomembranosa en paladar y lesión edematosa en punta de la lengua.

La figura 1 presenta la distribución de los pacientes del grupo I que desarrollaron complicaciones estomatológicas. Un total de 16 (40 \%) niños desarrollaron conjuntamente 61 episodios de tales complicaciones, la edad de éstos varió de los 2 meses a los 14 años, con media de 8 años, siendo 10 del sexo femenino y 6 del masculino.

El cuadro 2 muestra la distribución de los niños con sus respectivos episodios de candidiasis observados en el grupo I. Cinco niños (12.5\%), todos del sexo femenino, con edades de 2 a 12 años y media de 7,8 años, en tratamiento para neoplasias sistémicas desarrollaron en conjunto 9 episodios de ésta complicación estomatológica ( 6 de candidiasis pseudo- membranosa y 3 de queilitis angular) (Fig. 7). Mediante la aplicación del test exacto de Fisher no se observó diferencia estadísticamente significativa en la ocurrencia de candidiasis entre los niños con neoplasias sistémicas e aquellos con tumores sólidos $(\mathrm{p}=$ 0.13), sin embrago, estadísticamente, los niños con neoplasias sistémicas presentaron un riesgo mayor de desarrollar mucositis $(R R=0.80)($ Tabla 4).

El cuadro 3 muestra la distribución de los niños del grupo I que presentaron sangrado oral espontáneo, seis niños (15\%), siendo 5 del sexo femenino y 1 del masculino, con edades de 2 a 11 años y media de 8,1 años, todos en tratamiento para neoplasias sistémicas desarrollaron conjuntamente 10 episodios de sangrado oral espontáneo, de los cuales, 9 ocurrieron en encía y 1 en paladar duro, siendo éste último asociado a mucositis ulcerativa. Nueve episodios de sangrado oral espontáneo coincidieron con períodos en que los recuentos de plaquetas de los niños fueron menores que 100.000 céls $/ \mathrm{mm}^{3}$. Solo una niña de 10 años presentó sangrado oral espontáneo con recuento plaquetario de 473.000 céls $/ \mathrm{mm}^{3}$ (Cuadro 3). Estadísticamente el test exacto de Fisher no mostró diferencia estadísticamente significativa en la ocurrencia de sangrado oral espontáneo entre los niños con neoplasias sistémicas y aquellos con tumores sólidos ( $p=0.067$ ); sin embrago, fue observado que, estadísticamente los niños con neoplasias sistémicas tuvieron mayor riesgo de presentar sangrado oral espontáneo que los niños con tumores sólidos ( $R R=0.76)$ (Tabla 5). 
TABLA 4.- FRECUENCIA, VALOR DE P, RIESGO RELATIVO E INTERVALO DE CONFIANZA DE LOS NIÑOS CON CANDIDIASIS EN EL GRUPO I. NATAL / RN-2001

\begin{tabular}{|c|c|c|c|c|c|c|c|c|c|}
\hline \multicolumn{10}{|c|}{ Candidiasis } \\
\hline \multirow[b]{2}{*}{ Neoplasia } & \multicolumn{2}{|c|}{ Ausente } & \multicolumn{2}{|c|}{ presente } & \multicolumn{2}{|c|}{ Total } & \multirow{2}{*}{$p$} & \multirow{2}{*}{$R R$} & \multirow{2}{*}{ IC (95\%) } \\
\hline & $n$ & $\%$ & $n$ & $\%$ & $n$ & $\%$ & & & \\
\hline Neoplasias sistémicas & 20 & 80.0 & 5 & 20.0 & 25 & 62.5 & 0.13 & 0.80 & $0.65-0.97$ \\
\hline Tumores sólidos & 15 & 100 & 0 & 0 & 15 & 37.5 & & & \\
\hline Total & 35 & 87.5 & 5 & 12.5 & 40 & 100 & & & \\
\hline
\end{tabular}

\begin{tabular}{|c|c|c|c|c|c|}
\hline \multicolumn{6}{|c|}{$\begin{array}{l}\text { CUADRO 3.- DISTRIBUCIÓN DE LOS NIÑOS CON SUS RESPECTIVOS EPISODIOS DE } \\
\text { SANGRADO ORAL ESPONTÁNEO EN EL GRUPO I, SEGÚN LA EDAD, SEXO Y EL TIPO DE } \\
\text { NEOPLASIA. NATAL / RN-2001 }\end{array}$} \\
\hline Caso & Edad & Sexo & Neoplasia & Localización del sangrado & Recuento plaquetario \\
\hline 2 & $10 \mathrm{a}$ & $\mathrm{F}$ & LLA & Encía de los dientes 52, 5, 61 y 62 & $473.000 \mathrm{cel} / \mathrm{mm}^{3}$ \\
\hline 2 & $10 \mathrm{a}$ & $\mathrm{F}$ & LLA & Encía de los dientes 12 y 13 & $23.000 \mathrm{cel} / \mathrm{mm}^{3}$ \\
\hline 4 & $02 \mathrm{a}$ & M & LMA & Encía de los dientes 53, 54 y 55 & $28.000 \mathrm{cel} / \mathrm{mm}^{3}$ \\
\hline 17 & $10 \mathrm{a}$ & $\mathrm{F}$ & LMA & Encía de los dientes 64, 65, 26 & $26.000 \mathrm{cel} / \mathrm{mm}^{3}$ \\
\hline 18 & 09 a & $\mathrm{F}$ & LMA & Encía de los dientes 36 y 37 & $46.000 \mathrm{cel} / \mathrm{mm}^{3}$ \\
\hline 18 & 09 a & $\mathrm{F}$ & LMA & Encía de los dientes 35 y 36 & $83.000 \mathrm{cel} / \mathrm{mm}^{3}$ \\
\hline 22 & $07 \mathrm{a}$ & $\mathrm{F}$ & LMA & Encía de los dientes 74 y 75 & $25.000 \mathrm{cel} / \mathrm{mm}^{3}$ \\
\hline 22 & 07 a & $\mathrm{F}$ & LMA & $\begin{array}{l}\text { Paladar duro y encía adherida de los } \\
\text { molares superiores izquierdos }\end{array}$ & $40.000 \mathrm{cel} / \mathrm{mm}^{3}$ \\
\hline 22 & 07 a & $\mathrm{F}$ & LMA & Encía de los dientes 64 y 65 & $32.000 \mathrm{cel} / \mathrm{mm}^{3}$ \\
\hline 23 & $11 \mathrm{a}$ & $\mathrm{F}$ & LLA & $\begin{array}{l}\text { Encía de la región anterior de la } \\
\text { mandíbula }\end{array}$ & $99.000 \mathrm{cel} / \mathrm{mm}^{3}$ \\
\hline
\end{tabular}

TABLA 5.- FRECUENCIA, VALOR DE P, RIESGO RELATIVO E INTERVALO DE CONFIANZA DE LOS NIÑOS CON SANGRADO ORAL ESPONTÁNEO EN EL GRUPO I. NATAL / RN-2001

\begin{tabular}{|c|c|c|c|c|c|c|c|c|c|}
\hline \multicolumn{10}{|c|}{ Sangrado Oral Espontáneo } \\
\hline \multirow[b]{2}{*}{ Neoplasia } & \multicolumn{2}{|c|}{ Ausente } & \multicolumn{2}{|c|}{ presente } & \multicolumn{2}{|c|}{ Total } & \multirow{2}{*}{$p$} & \multirow{2}{*}{$R R$} & \multirow{2}{*}{ IC (95\%) } \\
\hline & $n$ & $\%$ & $n$ & $\%$ & $n$ & $\%$ & & & \\
\hline Neoplasias sistémicas & 19 & 76.0 & 6 & 24.0 & 25 & 62.5 & 0.067 & 0.76 & $0.61-0.76$ \\
\hline Tumores sólidos & 15 & 100 & 0 & 0 & 15 & 37.5 & & & \\
\hline Total & 34 & 85.0 & 6 & 15.0 & 40 & 100 & & & \\
\hline
\end{tabular}




\section{TABLA 6.- FRECUENCIA, VALOR DE P, RIESGO RELATIVO E INTERVALO DE CONFIANZA DE LOS NIÑOS CON MUCOSITIS EN EL GRUPO I. NATAL / RN-2001}

\begin{tabular}{|c|c|c|c|c|c|c|c|c|c|}
\hline \multicolumn{10}{|c|}{ Mucositis } \\
\hline \multirow[b]{2}{*}{ Neoplasia } & \multicolumn{2}{|c|}{ Ausente } & \multicolumn{2}{|c|}{ presente } & \multicolumn{2}{|c|}{ Total } & \multirow{2}{*}{$p$} & \multirow{2}{*}{$R R$} & \multirow{2}{*}{ IC (95\%) } \\
\hline & $n$ & $\%$ & $n$ & $\%$ & $n$ & $\%$ & & & \\
\hline Neoplasias sistémicas & 12 & 48.0 & 13 & 52.0 & 25 & 62.5 & 0.06 & 0.60 & $0.37-0.97$ \\
\hline Tumores sólidos & 12 & 80.0 & 3 & 20.0 & 15 & 37.5 & & & \\
\hline Total & 35 & 87.5 & 5 & 12.5 & 40 & 100 & & & \\
\hline
\end{tabular}

\begin{tabular}{|c|c|c|c|c|}
\hline \multicolumn{5}{|c|}{$\begin{array}{c}\text { CUADRO 4.- DISTRIBUCIÓN DE “OTRAS LESIONES” OBSERVADAS EN LOS NIÑOS DEL } \\
\text { GRUPO I, CON RELACIÓN A LA EDAD, SEXO Y TIPO DE NEOPLASIA. } \\
\text { NATAL / RN-2001 }\end{array}$} \\
\hline Caso & Edad & Sexo & Neoplasia & Lesiones \\
\hline 2 & $10 \mathrm{a}$ & $\mathrm{F}$ & LLA & Gingivitis, pericoronaritis. \\
\hline 5 & 03 a & M & Neuroblastoma & Gingivitis \\
\hline 9 & $12 \mathrm{a}$ & M & LLA & Aumento gingival, lesiones de "murcicatum labiuorum" \\
\hline 12 & 05 a & M & LLA & Úlcera traumática en mucosa de carrillos izquierda \\
\hline 17 & $10 \mathrm{a}$ & $\mathrm{F}$ & LMA & Gingivitis \\
\hline 18 & 09 a & $\mathrm{F}$ & LMA & Palidez de la mucosa \\
\hline 22 & 07 a & $\mathrm{F}$ & LMA & Absceso periapical \\
\hline 23 & $11 \mathrm{a}$ & $\mathrm{F}$ & LLA & Gingivitis, pericoronaritis \\
\hline 24 & 13 a & M & LMA & Gingivitis, petequia \\
\hline 25 & $04 \mathrm{a}$ & $\mathrm{F}$ & LLA & Úlcera traumática en lengua \\
\hline 30 & $02 \mathrm{a}$ & $M$ & LLA & $\begin{array}{l}\text { Úlcera traumática en labio inferior, abscesos periapicales en } \\
\text { dientes } 51 \text { y } 61\end{array}$ \\
\hline 31 & $12 \mathrm{a}$ & $\mathrm{F}$ & D. H. & Lengua vellosa, palidez de la mucosa \\
\hline 33 & $07 \mathrm{a}$ & $\mathrm{F}$ & LMA & Ulcera traumática, petequia \\
\hline 40 & $14 \mathrm{a}$ & $M$ & LLA & $\begin{array}{l}\text { Aumento gingival en región de dientes } 16 \text { y 46; aumento } \\
\text { de volumen en paladar duro. }\end{array}$ \\
\hline
\end{tabular}

Lesiones clínicamente diagnosticadas como mucositis oral ocurrieron en 13 (52 \%) niños del grupo I portadores de neoplasias malignas sistémicas. De éstos, 6 (46.2 \%) niños tenían LLA, 6 (46.2 \%) LMA y 1 (7.7 $\%$ ) enfermedad de Hodgkin. De tres niños (20 \%) con tumores sólidos, 2 (66.7 \%) que tenían neuroblastoma y 1 (33.3 \%) con fibrosarcoma, todos desarrollaron esa complicación estomatológica. Mediante la aplicación del test exacto de Fisher no fue observada diferencia estadísticamente significativa en la ocurrencia de mucositis entre los niños con neoplasias sistémicas y aquellos con tumores sólidos ( $\mathrm{p}=$ 0.06), a pesar de eso, estadísticamente los niños con neoplasias sistémicas presentaron un riesgo mayor de desarrollar mucositis que los niños con tumores sólidos $(\mathrm{RR}=0.60)$ (Tabla 6).

La mucositis ocurrió frecuentemente en la mucosa de los carrillos, labios, lengua, encía adherida, suelo 


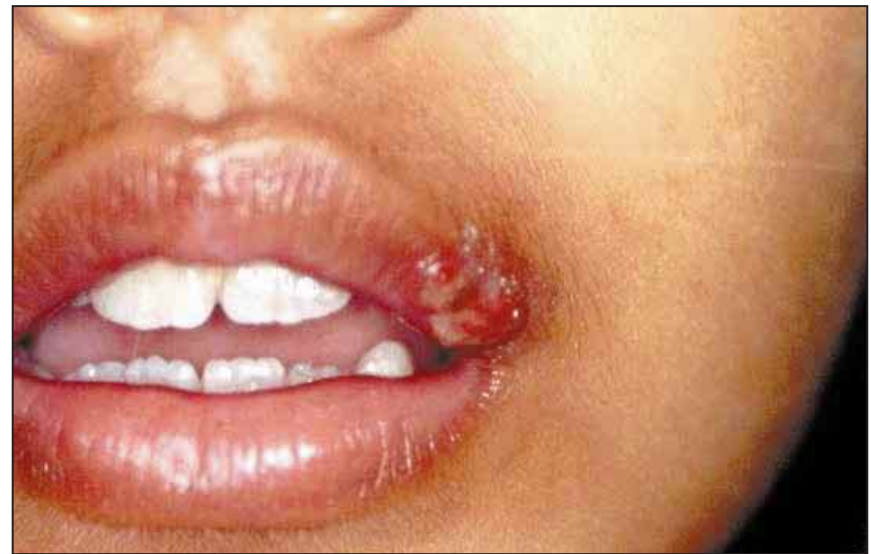

Figura 2. Paciente con LMA presentando mucositis en el labio superior. Aspecto inicial de la lesión caracterizado por eritema y edema.

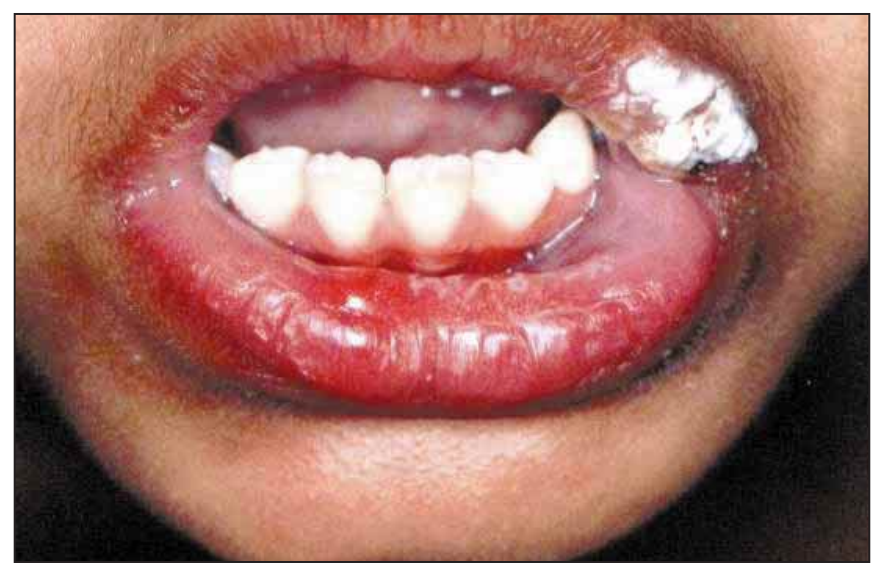

Figura 3. Paciente con LMA presentando lesión de mucositis en el labio superior, caracterizada por la presencia de ulceración y pseudomembrana blanca en la superficie (Mismo paciente de la Fig. 2)

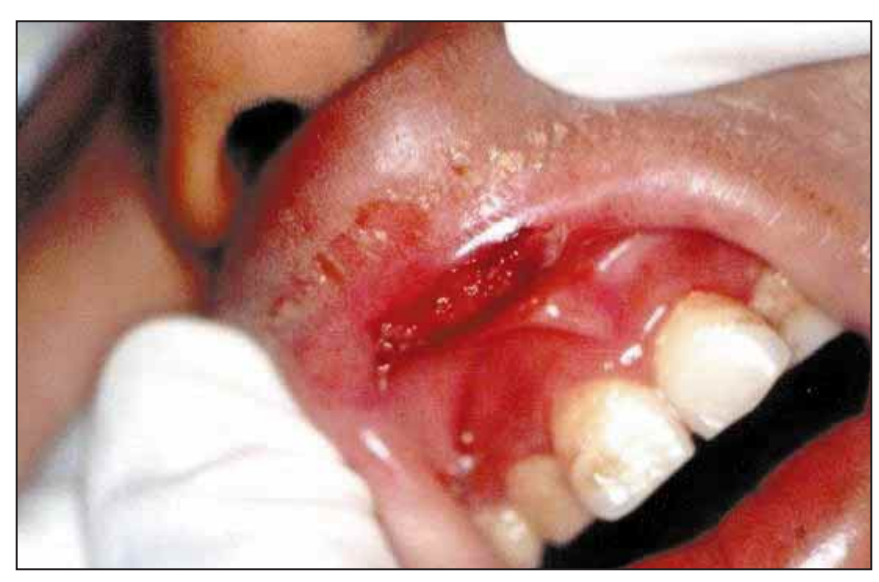

Figura 4. Paciente con LLA presentando lesión de mucositis en el lábio superior, caracterizada por la presencia de ulceración

de la boca y paladar duro (Figs. 2, 3, 4, 5 y 6). Una niña de 12 años con enfermedad de Hodgkin y un niño con fibrosarcoma desarrollaron xerostomia.

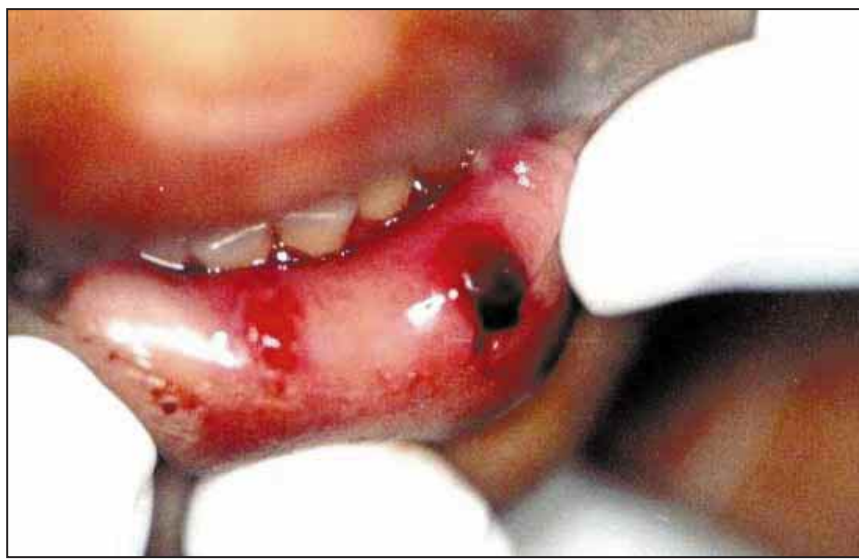

Figura 5. Paciente con LLA presentando mucositis en el labio inferior, caracterizada por eritema y ulceración.

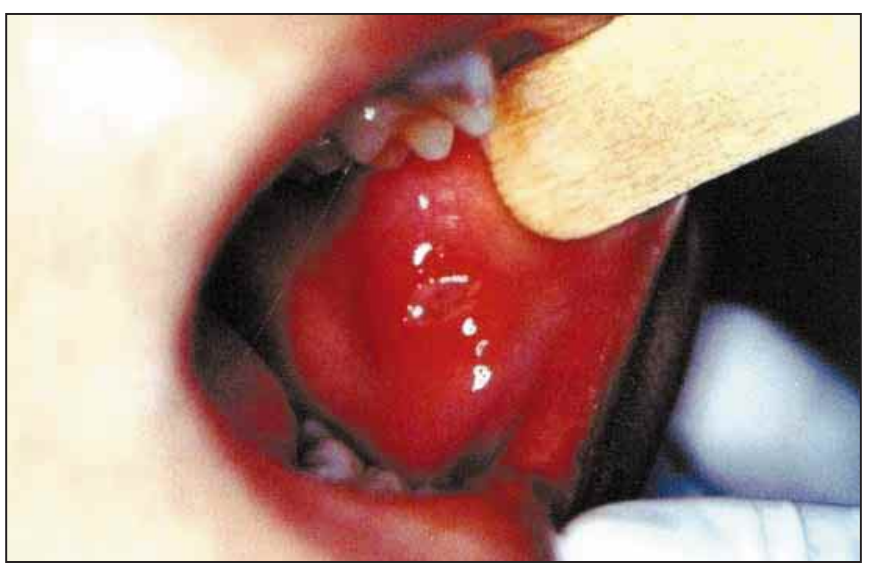

Figura 6. Paciente con LMA presentando mucositis en mucosa de carrillos, caracterizada por eritema y ulceración.

El cuadro 4 muestra la distribución de las lesiones observadas en 14 niños del grupo I que fueron consideradas como "otra lesiones". La mayoría de esos niños estaban en tratamiento para neoplasias sistémicas. En el grupo II, un paciente del sexo masculino, con 1 año de edad, presentó queilitis angular y otro del sexo masculino con 2 años de edad, presentó eritema migratorio benigno.

\section{DISCUSIÓN}

La mayoría de los canceres en la infancia tienen origen embrionario y aproximadamente la mitad afecta el sistema hematopoyético, recibiendo en éstos casos, la denominación de neoplasias sistémicas (13). Los otros tipos de neoplasias corresponden a los llamados tumores sólidos (2). 
En éste estudio, similarmente a lo relatado en la literatura, en el grupo I constituido por niños con neoplasias malignas, hubo predominancia de las neoplasias sistémicas, representadas por 25 casos (62.5\%), de los cuales, 21 eran leucemias agudas (13 LLA - 32.5 \% y 8 LMA - $20 \%$ ), contra 15 (37.5\%) casos de tumores sólidos. Éste hecho también fue constatado por Sonis y Kunz (1) que identificaron una predominancia de neoplasias sistémicas en 495 pacientes con diversos tipos de canceres.

Fue constatado que de los 13 casos de LLA, 11 (79.9 \%) ocurrieron en niños con 10 años de edad ó menores, resultados éstos similares a los obtenidos por Hou, Huang y Tsai (11), ya que, de 230 casos de leucemias, verificaron que $63.7 \%$ eran LLA en niños menores de 10 años de edad. Sonis y Kunz (1) también observaron una predominancia de leucemias agudas, con un total de 91 casos en 495 pacientes con diversos tipos de neoplasias malignas.

En éste estudio, de los 15 niños con tumores sólidos, 5 (33.3 \%) tenían neuroblastoma, 4 (26,7 \%) tumor de Wilms y 6 (40\%) sarcomas de tejidos blandos. Éstos resultados concuerdan con los datos de Grosfeld (2), que considera al neuroblastoma como el tumor sólido más común de la infancia, seguido del tumor de Wilms y de los sarcomas de tejidos blandos.

Debido a la mielosupresión causada por la neoplasia, ó por los efectos colaterales de la terapia antineoplásica, las complicaciones estomatológicas son consideradas de alto riesgo $(14,15)$. Las condiciones de salud oral también ejercen influencia en la ocurrencia de esas complicaciones $(6,16,17)$, una vez que, la boca constituye un ambiente favorable para la colonización y proliferación de microorganismos (18).

Los índices de placa visible (IPV) y de sangrado gingival (ISG), utilizados en éste estudio como parámetros para evaluar la salud bucal de los niños, no mostraron diferencias estadísticamente significativas entre los grupos I y II, a pesar de que el ISG haya sido mayor en el grupo II que mostró una condición de salud oral ligeramente más deficiente que los niños del grupo I. Éstos resultados, desde nuestra óptica, reflejan el producto de trabajos preventivos realizados anteriormente por Costa (19) y adoptados por el COHI del HIVS.
Los niños del grupo II presentaron mayor número de dientes cariados y estadísticamente fue observada diferencia estadísticamente significativa en el índice de CAO-D/cao-d entre los grupos I y II $(p=0.002$ ). Duggal y col. (16) obtuvieron resultados diferentes a los de éste estudio al comparar la salud oral de 46 pacientes con cáncer y 46 pacientes saludables, sin observar diferencia estadísticamente significativa entre los índices CAO-D/CAO-S de esos grupos, sin embargo el grupo con cáncer presentó mayor número de superficies dentárias cariadas y una prevalencia significativamente mayor de gingivitis.

Ciertas alteraciones orales pueden ser la primera señal del desarrollo de neoplasias malignas, principalmente, de las leucemias $(11,20)$. Esto fue constatado en éste estudio, ya que 2 niños desarrollaron lesiones orales que, junto a otras señales y síntomas, despertaron en los padres la sospecha de que algo anormal sucedía con sus hijos. Éste hecho motivó la atención médica que, después de los exámenes adecuados, emitió los diagnósticos de LMA y LLA respectivamente.

De 40 niños del grupo I, 16 (40\%) tuvieron conjuntamente 61 episodios de complicaciones estomatológicas comprendiendo mucositis, sangrado oral espontáneo, candidiasis y xerostomia. De esos pacientes, 6 estaban en tratamiento para LLA y 6 para LMA, presentando conjuntamente, 47 (87.03 $\%)$ de los episodios estomatológicos observados. Sonis y Kunz (1) constataron en un estudio de 495 pacientes con diferentes neoplasias malignas, que las neoplasias hematológicas mostraban mayor tendencia de generar complicaciones estomatológicas.

La mucositis oral es considerada la complicación más frecuente en pacientes con neoplasias malignas, estén éstos bajo radioterapia ó quimioterapia $(12,21,22$, $23,28)$. Los datos referentes a esa complicación en pacientes de éste estudio, coinciden con los de la literatura, pues la mucositis oral fue la complicación más frecuente en los niños del grupo I, ya que, 16 (40\%) de éstos, desarrollaron conjuntamente 40 episodios, que representaron $66 \%$ de todas las complicaciones estomatológicas registradas.

Driezen y cols. 24 obtuvieron resultados similares a los de éste estudio, ya que de 1500 pacientes con neoplasias malignas por ellos evaluados, 704 (46.9\%) desarrollaron complicaciones estomatológicas, resaltando 
el hecho que en 244 (16.3\%) de esos pacientes la complicación más frecuentes fue la mucositis oral.

En éste estudio fue constatado que la mucositis ocurrió principalmente en la mucosa de carrillos, labios, piso bucal y en regiones queratinizadas de la encía, lengua y paladar duro. Observamos que éstas lesiones ocurrían con mayor frecuencia en el periodo de 5 a 8 días después de iniciada la quimioterapia, persistiendo el cuadro clínico por 10 a 12 días. Éstos resultados coinciden con relatos existentes en la literatura (25).

Con relación al sangrado oral espontáneo, en el grupo I, seis $(15 \%)$ de los pacientes presentaron conjuntamente 10 episodios, representando $16.4 \%$ de las complicaciones estomatológicas observadas en éste grupo, estando todos esos niños bajo tratamiento para neoplasias sistémicas (4 LMA y 2 LLA). Fue detectado que $90 \%$ de los episodios de sangrado oral coincidieron con periodos en que los niños presentaban recuentos plaquetários menores que 100.000 $\mathrm{cel} / \mathrm{mm}^{3}$. Siete episodios (77.8\%) ocurrieron con conteos entre 23.000 y $46.000 \mathrm{cel} / \mathrm{mm}^{3}$, en dos episodios (22.2\%) los recuentos plaquetarios estuvieron entre 50.000 y $100.000 \mathrm{cel} / \mathrm{mm}^{3}$, sin embargo, a niña (10 $\%)$ mostró sangrado con recuento de 473.000 $\mathrm{cel} / \mathrm{mm}^{3}$. Éstos resultados también coinciden con los obtenidos por Hou, Huang y Tsai (11), los cuales consideraron recuentos plaquetarios entre 25.000 $\mathrm{cel} / \mathrm{mm}^{3}$ y $60.000 \mathrm{cel} / \mathrm{mm}^{3}$ como factor de riesgo para la ocurrencia de sangrado oral. Éstos autores observaron que de 83 casos de ésta complicación evaluados, 66 ocurrieron en asociación a recuentos plaquetarios menores que $60.000 \mathrm{cel} / \mathrm{mm}^{3}$. Es importante considerar que, según Fonseca (4) y McKenna (5), otros factores además de la trombocitopenia deben ser considerados con relación al sangrado oral, tales como, alteraciones cualitativas de las plaquetas ó de la coagulación. Probablemente, algunas de esas alteraciones podría haber provocado el sangrado espontáneo en éstos niños, principalmente en el que presentó recuento plaquetário de $473.000 \mathrm{cel} / \mathrm{mm}^{3}$.

De los 10 casos de sangrado oral espontáneo, $90 \%$ ocurrieron en encía y 10 \% fueron asociados a mucositis ulcerativa en paladar duro. Driezen y cols.(24) detectaron en su estudio que de 1.500 pacientes con leucemias, 204 presentaron sangrado oral como consecuencia de diversas causas.
En éste estudio, fue posible observar el desarrollo de 9 lesiones clínicamente diagnosticadas como candidiasis, distribuidas en 5 pacientes del grupo I. La candidiasis pseudomembranosa fue la que predominó con $66.7 \%$ de los episodios, prevaleciendo en las regiones de la mucosa de carrillos, lengua, suelo de la boca, encía adherida, además de 3 casos (33.3 \%) de queilitis angular. Éstos resultados reflejan el hecho que la candidiasis representa aproximadamente, $50 \%$ de las infecciones orales en pacientes inmunológicamente comprometidos, principalmente, la de tipo pseudomembranosa, acometiendo las regiones observadas en éste estudio $(6,26,27)$.

Con relación a la ocurrencia de xerostomia, ésta fue mínima siendo, constatado solo el comprometimiento de $2(5 \%)$ niños del grupo I en tratamiento para fibrosarcoma y enfermedad de Hodgkin respectivamente; solo éste último paciente había recibido radioterapia. Probablemente la poca ocurrencia de xerostomia podría estar relacionada a la baja dosis de radiación utilizada, que no fue capaz de inducir ésta complicación, ó al hecho de que la radiación fue practicada en ese paciente al final del período de nuestras evaluaciones clínicas y posiblemente no había pasado el tiempo necesario para que sus efectos colaterales pudieran ser evidenciados clínicamente en la boca.

En el grupo I fue observado que 14 niños presentaron conjuntamente 22 alteraciones orales que fueron clasificadas como "otras lesiones", predominando entre ellas, 5 casos de gingivitis y 4 casos de úlcera traumática. A pesar de que los niños del grupo II presentaron una condición de salud oral más pobre que los del grupo I, como se esperaba, éstos no presentaron alteraciones orales además de las ya mencionadas en ésta discusión.

Es importante resaltar que la mayoría de los niños del grupo I que desarrollaron las diversas complicaciones estomatológicas constatadas en éste estudio correspondieron precisamente a aquellos que presentaron mayores IPV, ISG e CAO-D/cao-d, ó sea, condiciones deficientes de higiene oral; ésto no estaría relacionado obligatoriamente a la falta de conocimientos de higiene oral, ya que en ambos grupos los niños, así como sus padres y/ó acompañantes fueron periódicamente orientados sobre cuidados de higiene oral y sensibilizados sobre la importancia de los mismos para evitar 
y/ó minimizar la ocurrencia de complicaciones estomatológicas. Creemos que el propio hecho de la presencia de las complicaciones estomatológicas actuaba como factor adverso para que los niños se rehusaran a realizar la higiene bucal, presentando así condiciones de salud oral deficientes y consiguientemente mayor riesgo de desarrollar esas complicaciones.

En los estudios de Sepet y cols.(17) sobre la salud oral de 41 niños con LLA y 20 sanos, fue posible observar que, a pesar de que la frecuencia de visitas al dentista y los cuidados de cepillado fueron similares en los dos grupos por ellos evaluados, el patrón de higiene oral fue deficiente en los pacientes con LLA.

Concluyese con éste estudio que los pacientes bajo tratamiento para neoplasias malignas, principalmente los portadores de neoplasias sistémicas, presentan alto riesgo de desarrollar complicaciones estomatológicas, sobresaliendo entre ellas la mucositis y que ese riesgo se torna mayor cuando las condiciones de salud e higiene oral son deficientes.

Nuevos estudios, evaluando una muestra mayor y durante un periodo de tiempo relativamente grande son necesarios para observar clínicamente las complicaciones orales que los pacientes pediátricos con cáncer pueden desarrollar, haciendo énfasis en la relación de esas complicaciones con las condiciones de higiene y salud bucal.

\section{REFERENCIAS BIBLIOGRÁFICAS}

1. Sonis S T, Kunz A. Impact of improved dental services in the frequency of oral complications of cancer therapy for patients with non-head-and-neck malignancies. Oral Surg Oral Med Oral Pathol 1988; 65(1): 19-22.

2. Grosfeld J L. Risk-based management: Current concepts of treating malignant solid tumors in childhood. J Am Coll Surg 1999; 189(4): 407-25.

3. Barrett A P. A long-term prospective clinical study of oral complications during conventional chemotherapy for acute leukemia. Oral Surg Oral Med Oral Pathol 1987; 77: 13-9.

4. Fonseca M A. Pediatric bone marrow transplan- tation: Oral complications and recommendations for care. Pediatric Dentistry 1998; 20(7): 386-94.

5. McKenna S J. Leukemia. Oral Surg Oral Med Oral Pathol Oral Radiol Endod 2000; 89(2): 137-39.

6. Peterson D E, D'Ambrosio J A. Diagnosis and management of acute and chronic oral complications of nonsurgical cancer therapies. Dental Clinics of North America 1992; 36(4): 945-66.

7. Lang N P. Commonly used indices to assess oral hygiene and gingival and periodontal health and diseases. En: Lang N P, Attström R, Löe $H$. Proceeding of the European workshop on mechanical plaque control: status of the art and science of dental plaque control. Chicago: Quintessence, 1998.

8. Maltz M, Carvalho J. Diagnóstico da Doença Carie. En: Promoção de Saúde Bucal - ABOPREV. São Paulo: Artes Médicas, 1997:69-91.

9. Opperman R V, Rosing C K. Prevenção e Tratamento das Doenças Periodontais. En: Promoção de Saúde Bucal - ABOPREV. São Paulo: Artes Médicas, 1997:255-81

10. EC-CLEARINGHOUSE ON ORAL PROBLEMS RELATED TO HIV INFECTION AND WHO COLABORATING CENTRE ON ORAL MANIFESTATIONS OF THE IMMUNODEFICIENCY VIRUS. Classification and diagnostic criteria for oral lesions in HIV infection. J Oral Pathol Med 1993; 22(7): 289-91.

11. Hou G L, Huang J S, Tsai C C. Analysis of oral manifestations of leukemia: a retrospective study. Oral Diseases 1997; 3(1):31-8.

12. Epstein J B, Gorsky M, Guglietta A, Le N, Sonis S T. The correlation between epidermal growth factor levels in saliva and the severity of oral mucositis during oropharingeal radiation therapy. Cancer 2000; 89(11): 2258-65.

13. INCA: Particularidades do câncer infantil. [site de la internet] Disponible en: http://www.inca.org.br/ cancer/tipos.html. Accesado: 31 / enero / 2001.

14. Alpaslan G, Alpaslan C, Gogen H, Oguz A, Cetiner S, Karadeniz C. Disturbances in oral and dental structures in patients with pediatric lymphoma after 
chemotherapy. Oral Surg Oral Med Oral Pathol Oral Radiol Endod 1999; 87(3): 317-21.

15. Caielli C, Martha P M, Dib L L. Seqüelas orais da radioterapia: atuação da odontologia na prevenção e tratamento. Revista Brasileira de Cancerologia 1995; 41(4): $231-41$.

16. Duggal M S, Curzon M E J, Bailey C C, Lewis I J, Prendergast $M$. Dental parameters in long term survivors of childhood cancer compared with siblings. Oral Oncology 1997; 33(5): 348-53.

17. Sepet E, Aytepe Z, Ozerkan A G y cols. Acute lymphoblastic leukemia: dental health of children in maintenance therapy. J Clin Pediatr Dent 1998; 22(3): 257-60.

18. Sixou J L, Medeiros-Batista O, Bonaure-Mallet M. Modifications of the microflora of the cavity arising during immunosuppressive chemotherapy. Oral Oncology, Eur J Cancer 1996; 32B(5): 306-10.

19. Costa E M. Aspectos preventivos da clorexidina sobre as alterações da mucosa oral de crianças leucêmicas submetidas à quimioterapia. [Dissertação de Mestrado em Patologia Oral]. Nata (RN): Universidade Federal do Rio Grande do Norte, 1998.

20. Orbak R, Orbak Z. Oral condition of patient with leukemia and lymphoma. J Nihon Univ Sch Dent 1997; 39(2): 67-70.

21. Childers N K, Steinnett E A, Wheeler P, Wrigth J T, Castleberry R P, Dasanayaki A P. Oral complications in children with cancer. Oral Surg Oral Med Pathol Oral 1993; 75(1): 41-7.

22. Scully C, Epstein J B. Oral health care for the cancer patients. Oral Oncology, Eur J. Cancer 1996; 22B(5): 281-292.
23. Plevová P. Prevention and treatment of chemotherapy and radiotherapy-induced oral mucositis: a review. Oral Oncology 1999; 35: 453-70.

24. Driezen S, McCredie K, Bodey G P, Keating M J. Quantitative analysis of the oral complications of antileukemia chemotherapy. Oral Surg Oral Med Oral Pathol 1986; 62(6): 650-53.

25. Woo S B, Sonis S T, Monopoli M M, Sonis A L. A longitudinal study of oral ulcerative mucositis in bone marrow transplant recipient. Cancer 1993; 72(5): 1612-17.

26. National Institute of Health Consensus Development Conference Statement: Oral complications of cancer therapies: diagnosis, preventions, and treatment. JADA 1989; 119: 231-241.

27. Flaitz CM, Baker K A. Treatment approaches to common symptomatic oral lesions in children. Dental Clinics of North America 2000; 44(3): 67196.

28. Sonis S T. Mucositis as a biological process: a new hypothesis for the development of chemotherapyinduced stomatotoxicity. Oral Oncology 1998; 34(1): 39-43.

\section{CORRESPONDENCIA}

Manuel Antonio Gordón-Núñez

Programa de Pós-Graduação em Patologia Oral UFRN

Avenida Senador Salgado Filho, 1787.

Bairro Lagoa Nova - Natal / RN

CEP: 59056-000.

Fone / Fax: (084) 215-4138

Brasil 